\title{
Minimally invasive lateral interbody fusion for the treatment of rostral adjacent-segment lumbar degenerative stenosis without supplemental pedicle screw fixation
}

\author{
Clinical article
}

\author{
Michael Y. Wang, M.D., ${ }^{1}$ Ram Vasudevan, M.D., ${ }^{2}$ and Stefan A. Mindea, M.D. ${ }^{2}$ \\ ${ }^{1}$ Department of Neurological Surgery, University of Miami Miller School of Medicine, Miami, Florida; and \\ ${ }^{2}$ Department of Neurological Surgery, Stanford University School of Medicine, Stanford, California
}

\begin{abstract}
Object. Adjacent-segment degeneration and stenosis are common in patients who have undergone previous lumbar fusion. Treatment typically involves a revision posterior approach, which requires management of postoperative scar tissue and previously implanted instrumentation. A minimally invasive lateral approach allows the surgeon to potentially reduce the risk of these hazards. The technique relies on indirect decompression to treat central and foraminal stenosis and placement of a graft with a large surface area to promote robust fusion and stability in concert with the surrounding tensioned ligaments. The goal in this study was to determine if lateral interbody fusion without supplemental pedicle screws is effective in treating adjacent-segment disease.

Methods. For a 30-month study period at two institutions, the authors obtained all cases of lumbar fusion with new back and leg pain due to adjacent-segment stenosis and spondylosis failing conservative measures. All patients had undergone minimally invasive lateral interbody fusion from the side of greater leg pain without supplemental pedicle screw fixation. Patients were excluded from the study if they had undergone surgery for a nondegenerative etiology such as infection or trauma. They were also excluded if the intervention involved supplemental posterior instrumented fusion with transpedicular screws. Postoperative metrics included numeric pain scale (NPS) scores for leg and back pain. All patients underwent dynamic radiographs and CT scanning to assess stability and fusion after surgery.

Results. During the 30-month study period, 21 patients (43\% female) were successfully treated using minimally invasive lateral interbody fusion without the need for subsequent posterior transpedicular fixation. The mean patient age was 61 years (range 37-87 years). Four patients had two adjacent levels fused, while the remainder had singlelevel surgery. All patients underwent surgery without conversion to a traditional open technique, and recombinant human bone morphogenetic protein-2 was used in the interbody space in all cases. The mean follow-up was 23.6 months. The mean operative time was 86 minutes, and the mean blood loss was $93 \mathrm{ml}$. There were no major intraoperative complications, but one patient underwent subsequent direct decompression in a delayed fashion. The leg pain NPS score improved from a mean of 6.3 to $1.9(\mathrm{p}<0.01)$, and the back pain NPS score improved from a mean of 7.5 to $2.9(\mathrm{p}<0.01)$. Intervertebral settling averaged $1.7 \mathrm{~mm}$. All patients had bridging bone on CT scanning at the last follow-up, indicating solid bony fusion.

Conclusions. Adjacent-segment stenosis and spondylosis can be treated with a number of different operative techniques. Lateral interbody fusion provides an attractive alternative with reduced blood loss and complications, as there is no need to re-explore a previous laminectomy site. In this limited series a minimally invasive lateral approach provided high fusion rates when performed with osteobiological adjuvants. (http://thejns.org/doi/abs/10.3171/2014.8.SPINE13841)
\end{abstract}

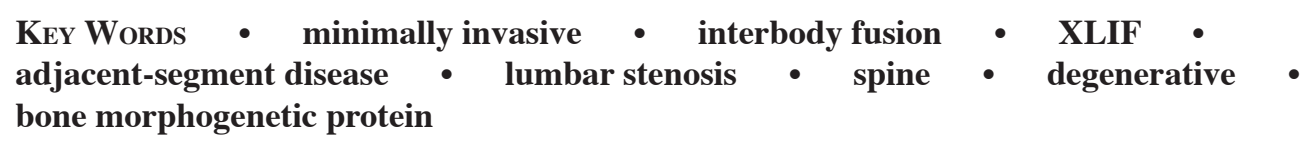

A DJACENT-segment disease (ASD) is common following lumbar spinal fusion. While some authors have theorized that this is the result of increased biomechanical forces on the neighboring motion seg-

Abbreviations used in this paper: ALIF = anterior lumbar interbody fusion; ASD = adjacent-segment disease; $\mathrm{BMP}=$ bone morphogenetic protein; NPS = numeric pain scale; rhBMP-2 = recombinant human BMP-2. ments, it is also clear that the unique biological and environmental factors for a patient who has already required a previous lumbar fusion predisposes that individual to additional degeneration at other levels of the spinal column. The patient developing ASD may present with axial pain due to spondylosis and/or lower extremity symptoms due to stenosis or nerve root entrapment.

For the surgeon treating ASD, a number of treatment options are available. For cases involving only neural en- 
trapment without axial symptoms, the surgeon may opt for limited decompression. However, this approach risks iatrogenic destabilization in the setting of advanced spondylosis. A more typical approach is to perform revision posterior surgery with both a laminectomy and extension of the instrumentation and fusion to the rostral level(s). However, this method requires extensive soft tissue dissection to expose the previously implanted hardware, adding to surgical blood loss and postoperative pain and prolonging recovery with high associated health care costs..$^{13}$ For example, Smorqick et al. found that revision posterior surgery involved a mean blood loss of 1606 $\mathrm{ml}, 16 \%$ greater than in similar primary surgeries. ${ }^{15}$ The surgeon will typically have to explant or attach onto the previous hardware construct, expanding the surgical enterprise. In addition, exposing the previous laminectomy site poses a higher risk of dural violations and CSF leakage due to postoperative scar tissue. ${ }^{9}$

An alternative strategy for revision surgery is to opt for an entirely different access route to the spine. Minimally invasive surgical techniques to fuse and decompress the spinal column have become increasingly popular over the past decade. In particular, the minimally invasive lateral approach innovated by Pimenta has become popular given the advent of electrophysiological navigation and tubular retractor technology. ${ }^{5,7,12}$ These developments have allowed for less invasive access to the spinal column by navigating through the lumbosacral plexus and psoas muscle using real-time electromyographic feedback. ${ }^{16}$ A lateral approach also gives the surgeon the ability to achieve a robust fusion by using a cage that spans the vertebral endplates from the right to the left side. This places the cage in contact with the apophyseal ring of the vertebral body, which is the strongest area of the endplate, and reduces the likelihood of settling as fusion occurs. Preservation of the anterior and posterior longitudinal ligaments allows for tensioning of these structures to confer stability using the cage alone.

The wide application of anterior and anterolateral interbody fusions has also led to the realization that indirect decompression can be useful for treating neurological compression. Indirect decompression relies on distraction across the intervertebral space to stretch the spinal ligaments and open the central canal as well as increase the foraminal space for the exiting nerve root. This approach has since been validated as a reliable method of increasing the foraminal space. In a study by Cho et al., anterior lumbar interbody fusion (ALIF) increased foraminal space by an average of $43 \% .^{3}$ A study of lateral interbody fusion by Kepler et al. showed a $35 \%$ increase of foraminal space. ${ }^{8}$ Elowitz et al. showed that in 15 patients dural sac dimensions in the anteroposterior and mediolateral planes improved by $54 \%$ and $48 \%$, respectively. ${ }^{6}$ The dural sac area increased an average of $143 \%$.

In the present study we explored a small series of patients who had undergone minimally invasive lateral interbody fusion to achieve indirect decompression and stabilization for ASD without supplemental pedicle screw fixation. This method has been controversial given concerns that fusion rates may suffer due to the stiff caudal instrumented construct. However, growing experience with anecdotal cases indicates that a lateral minimally invasive approach may indeed be acceptable for limited rostral fusions.

\section{Methods}

\section{Patient Population}

Cases were retrospectively evaluated under institutional review board exemptions. For a 30-month period at two academic institutions, we sought cases of low lumbar fusion in which clinical and radiographic findings were consistent with progressive degeneration at the adjacent spinal level(s) with associated new back and/or leg symptoms failing conservative measures, including physical therapy, antiinflammatory medications, low-dose narcotics, and epidural injections. Presentations included neurogenic claudication, radiculopathy, and/or intractable low-back pain. All patients had undergone minimally invasive lateral interbody fusion from the side of greater leg pain without supplemental pedicle screw fixation. Patients were excluded from the study if they had undergone surgery for a nondegenerative etiology such as infection or trauma. They were also excluded if the intervention involved supplemental posterior instrumented fusion with transpedicular screws.

\section{Surgical Technique}

The surgery is accomplished with the patient in the lateral position while under general anesthesia. Positioning in the lateral position is critical to flex the spine to open the side of the approach, spreading the space between the lower rib cage and the iliac crest. A $3-\mathrm{cm}$ transverse flank incision is made directly over the disc space(s) of interest, and the muscular layers are dissected to expose the retroperitoneal space. The psoas muscle belly is identified, and a Kirschner wire (K-wire) is placed into the disc space of interest under lateral fluoroscopic imaging. In some cases a second smaller incision is made more posteriorly to dissect the retroperitoneal space bluntly with a finger. The wire is passed with the surgeon's finger guiding it to ensure safe access without violation of any vascular or hollow viscus structures. A succession of tubular retractors is passed over the K-wire, allowing placement of a final expandable retractor; this is done under live electromyography surveillance. The surface of the psoas muscle is mapped to ensure that there are no lumbosacral nerves in proximity. The route of access is confirmed on anteroposterior imaging, and the remainder of the discectomy, fusion, and hardware placement is performed under anteroposterior fluoroscopy.

After incising the annulus, we use curettes and rongeurs to remove the disc material. The vertebral endplates are prepared, taking care not to violate the cortical endplates to minimize cage settling. Care is also taken to ensure that the contralateral annulus is opened to allow for proper intervertebral distraction. The anterior and posterior longitudinal ligaments are preserved to allow tensioning of these structures, with cage distraction to improve structural rigidity and allow for indirect neural decompression.

Cage height is chosen based on preoperative disc 


\section{Minimally invasive lateral fusion for adjacent disease}

height, mobility of the motion segment with intraoperative manipulation, bone density, and degree of indirect decompression needed. An appropriately sized polyetheretherketone (PEEK) or carbon fiber cage is then filled with two recombinant human bone morphogenetic protein-2 (rhBMP-2) sponges (InFuse, Medtronic Sofamor Danek) for a dose of $4.2 \mathrm{mg} /$ level. Note that this is an off-label use of rhBMP-2 based on FDA clearance. After surgery, patients are kept in the hospital until they are able to pass flatus, eat, void, and ambulate independently. All patients wear an external orthosis for 12 weeks.

\section{Outcome Measures}

Clinical metrics included preoperative and postoperative numeric pain scale (NPS) scores obtained separately for leg and back pain. Scoring was performed at routine postoperative clinic visits and determined using a 10 -point scale, with 10 being the greatest pain and 0 being the absence of pain. Radiographic analysis was performed on dynamic radiographs to assess stability and fusion after surgery. Intervertebral height was measured (in $\mathrm{mm}$ ) by comparing the mid-disc height from one adjacent endplate to the next across the fusion site. Settling was then determined as the difference between immediate postoperative and last follow-up intervertebral heights. Segmental lordosis was determined by measuring the sagittal Cobb angle between the upper endplate of the upper vertebral body in relation to the lower endplate of the lower vertebral body fused. All patients underwent spiral slice CT scanning with sagittal and coronal reconstructed images to assess bridging bone to determine if bony fusion had occurred. Fusion was confirmed with the radiologist's official reading of the CT scan. Statistical comparisons were determined using a Student t-test.

\section{Results}

\section{Case Series}

During the study period, 21 consecutive patients were successfully treated at 25 levels (Figs. 1 and 2). There were no conversions to open surgery and no aborted procedures (Table 1). Neither was there any case in which an unplanned supplemental posterior pedicle screw had to be placed. The mean patient age was 61 years (range $37-87$ years), and $43 \%$ of the patients were female. Four of the patients had 2 levels fused, while the remainder had single-level surgery. The prior surgery had been performed for a mean of $2.3 \pm 0.80$ fusion levels (range 1-3 levels). Surgery was performed at the L1-2 level (1 case), the L2-3 level (13 cases), the L3-4 level (10 cases), and the L4-5 level (1 case). The rhBMP-2 was used in the interbody space in all cases. Cage height varied from 8 to $14 \mathrm{~mm}$, with a mean height of $10.3 \mathrm{~mm}$. The mean followup was 23.6 months, with all patients continuing with outpatient visits until radiographic fusion was confirmed on CT scanning and clinical outcomes were stabilized.

The mean total operative time was $86.2 \pm 29.5 \mathrm{~min}-$ utes (range 45-155 minutes), and mean blood loss was $93.1 \pm 55.1 \mathrm{ml}$ (range 20-200 ml). In 6 patients, anterior vertebral body screws had been placed or anterolateral plating had been accomplished at the time of the index surgery. The inpatient duration of stay averaged $2.4 \pm$ 0.97 days (range $1-4$ days). All patients were discharged to home.

\section{Outcomes and Complications}

The leg pain NPS score improved from a mean of 6.3 to 1.9 ( $\mathrm{p}<0.01$ ), and the back pain NPS score improved from a mean of 7.5 to 2.9 ( $\mathrm{p}<0.01$ ). Two of the patients experienced no clinical improvement in one of the pain measures (Table 2). One patient had symptoms primarily of neurogenic claudication. Treatment with 2-level surgery at L2-4 resulted in minimal indirect decompression as seen on postoperative myelography. Although the symptoms of back pain improved, the patient continued to experience distance-limited gait. He later underwent direct decompression for failure of indirect decompression using the cage to restore neurological function. However, even after the direct decompression procedure (hemilaminotomy with medial facetectomy at L2-3 and L3-4), he did not experience relief. Another patient had severe mechanical low-back pain. Surgery improved his leg pain, which was minimal, but his back pain remained 9 out of 10 . It was determined to be primarily due to sacroiliac joint arthritis based on a positive response to repeated injections.

Immediate postoperative intervertebral height was $10.4 \pm 1.4 \mathrm{~mm}$. At the last follow-up, intervertebral height reduced to an average of $8.7 \pm 1.6 \mathrm{~mm}$, indicating intervertebral settling of $1.7 \mathrm{~mm}$. Segmental lordosis improved from a mean of $9.8^{\circ}$ to $11.9^{\circ}$ for each of the 25 levels between the preoperative and final follow-up radiographs. However, global lumbar lordosis (L1-S1) decreased by $2^{\circ}$ comparing preoperative and last follow-up radiographs $\left(44^{\circ} \pm 21^{\circ}\right.$ vs $\left.42^{\circ} \pm 19^{\circ}\right)$. All patients demonstrated bridging bone on CT scanning at the last follow-up, indicating solid bony fusion.

\section{Complications}

There were no major or minor intraoperative or perioperative complications. New thigh numbness, pain, dysesthesias, or weakness indicative of a lumbosacral plexopathy were not seen in any of the patients by their 3-month follow-up visit. Two patients had a more extended hospital stay because of worsened constipation postoperatively. However, there were no cases of hollow viscus injuries, vascular insults, hernias, wound infections, CSF leakage, or cosmetic problems. One patient had the index lateral surgery at L2-3, but then developed an adjacentlevel problem at L1-2. She underwent posterior revision surgery that crossed the thoracolumbar junction.

\section{Discussion}

Adjacent-level degeneration has been reported to occur with some regularity after lumbar fusion surgery. In a recent literature review by Radcliff et al., ${ }^{14}$ the rate of symptomatic ASD after decompression and stabilization procedures approximated $2 \%-3 \%$ per year. Given this high incidence, spinal surgeons are facing a growing population of patients in need of treatment for ASD. In 

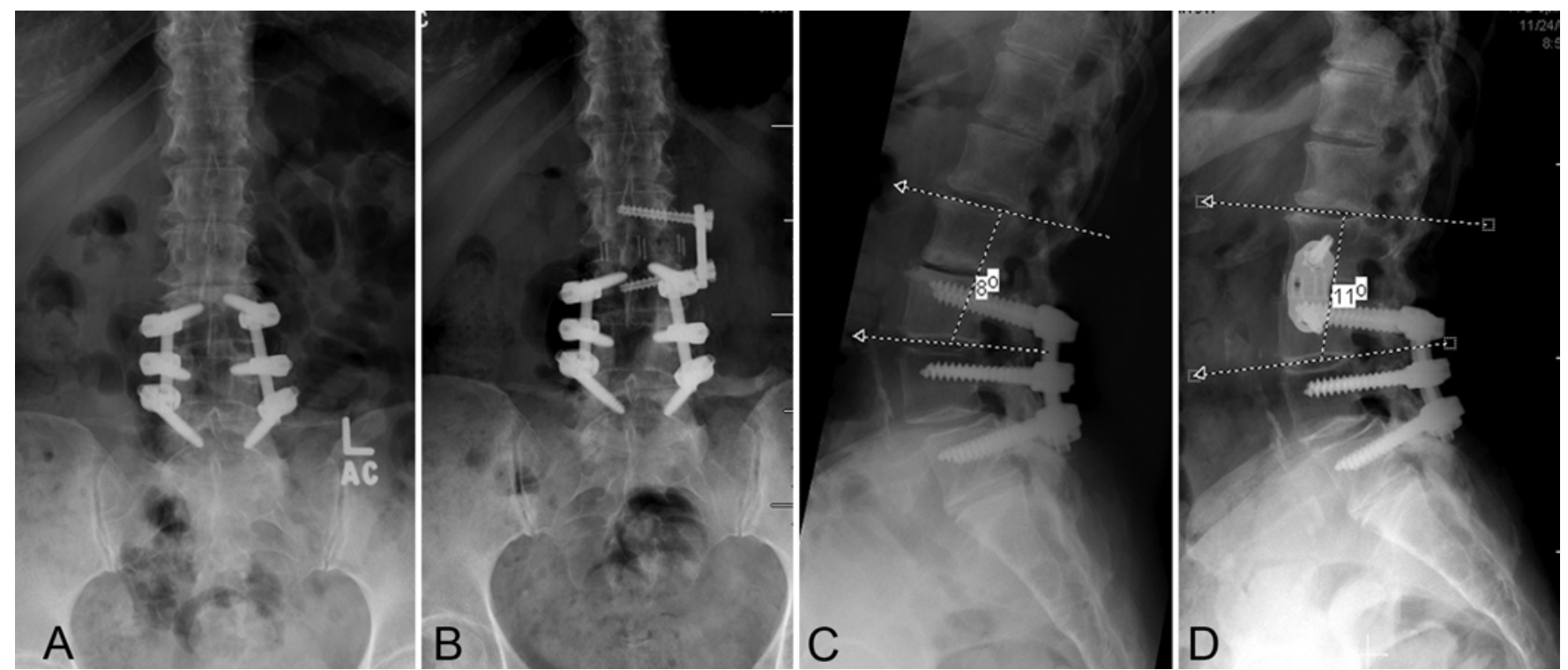

FIG. 1. Images obtained in a 57-year-old woman who had an L4-S1 instrumented fusion 6 years earlier. She experienced new intractable back and leg pain due to adjacent-segment degeneration and stenosis. A: Preoperative anteroposterior radiograph. B: Postoperative anteroposterior radiograph after a minimally invasive lateral interbody fusion with plating. C and D: Measurement of preoperative and postoperative segmental lordosis angle.

this series we treated patients with rostral ASD following a previous lumbar or lumbosacral fusion. Using rhBMP-2 and synthetic cages, we identified a high fusion rate that was associated with a concomitant improvement in clinical signs and symptoms of both back and leg pain. These findings suggest that supplemental posterior instrumentation does not need to be performed for all cases of ASD treated surgically.

A lateral approach is particularly suited for treating lumbar ASD since the majority of primary lumbar fusions occur at the L3-S1 level. Most ASD occurs proximal to this location and thus would involve surgery at the mid or high lumbar segments. This reduces the potential for problems with neurological complications associated with lateral transpsoas surgery at the L4-5 level. ${ }^{2}$ Indeed, in the present limited case series, we identified a very low
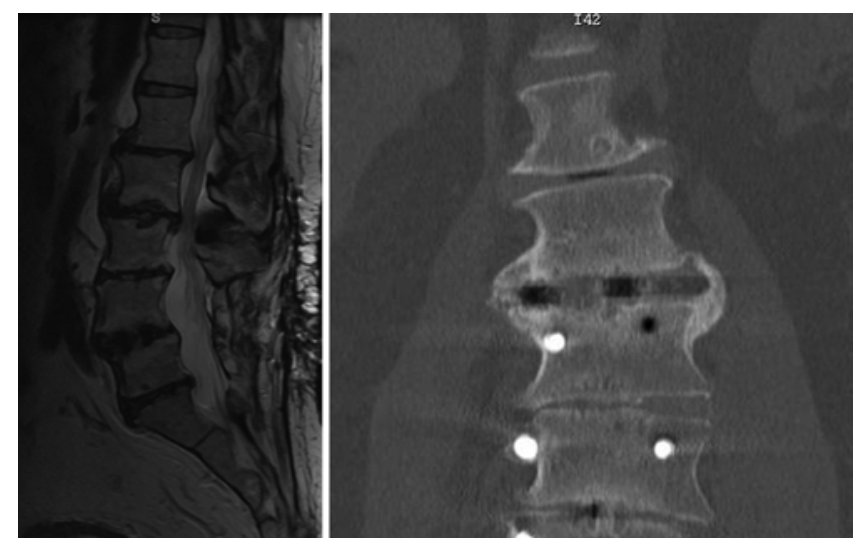

FIG. 2. Images obtained in a 66-year-old man who had previously undergone an L3-5 posterior instrumented fusion. Left: Lateral interbody fusion was performed for adjacent-level disc degeneration with herniation and stenosis. Right: Coronal reconstruction CT showing bony fusion across the L2-3 disc space within and around the intervertebral cage. incidence of new thigh pain, weakness, numbness, or dysesthesias compared with that in a previous series. ${ }^{4}$ This is probably attributable to the fact that the majority of cases involved levels at or above the L-3 exiting nerve root.

This approach has the advantage of being minimally invasive. Using only a small flank incision, we were able to

\section{TABLE 1: Summary of patient demographics and surgical} characteristics

\begin{tabular}{lc}
\hline \multicolumn{1}{c}{ Parameter } & Value \\
\hline total no. of patients & 21 \\
mean age in yrs & $61 \pm 12$ \\
age range in yrs & $37-87$ \\
sex ratio (M:F) & $12: 9$ \\
patients w/ 1-level surgery & $81 \%$ \\
patients w/ 2-level surgery & $19 \%$ \\
level of treatment & \\
$\quad$ L1-2 & 1 \\
$\quad$ L2-3 & 13 \\
$\quad$ L3-4 & 10 \\
$\quad$ L4-5 & 1 \\
total no. of levels treated & 25 \\
mean no. of previous caudal fusion levels & $2.3 \pm 0.8$ \\
mean cage height in mm & 10.3 \\
cage height range in mm & $8-14$ \\
use of rhBMP-2 & $100 \%$ \\
mean op time in mins & $86.2 \pm 29.5$ \\
op time range in mins & $45-155$ \\
mean blood loss in ml & $93.1 \pm 55.1$ \\
blood loss range in ml & $20-200$ \\
mean follow-up in mos & 23.6 \\
\hline
\end{tabular}


Minimally invasive lateral fusion for adjacent disease

TABLE 2: Clinical and radiographic outcomes

\begin{tabular}{lc}
\hline \multicolumn{1}{c}{ Parameter } & Value \\
\hline preop NPS, leg & $6.3 \pm 2.1$ \\
postop NPS, leg & $1.9 \pm 2.1$ \\
preop NPS, back & $7.5 \pm 1.9$ \\
postop NPS, back & $2.9 \pm 2.6$ \\
mean hospital stay in days & $2.4 \pm 0.97$ \\
length of stay range in days & $1-4$ \\
patients discharged to home & $100 \%$ \\
fusion rate (CT confirmation) & $100 \%$ \\
mean intervertebral settling in mm & 1.7 \\
neural decompression revision rate & $4.5 \%$ \\
ASD revision rate & $4.5 \%$ \\
intervertebral height in mm & \\
preop & $10.4 \pm 1.4$ \\
last follow-up & $8.7 \pm 1.6$ \\
global lumbar lordosis (L1-S1) & \\
preop & $44^{\circ} \pm 21^{\circ}$ \\
last follow-up & $42^{\circ} \pm 19^{\circ}$ \\
regional lumbar lordosis (at treated level) & \\
preop & $9.8^{\circ} \pm 3.2^{\circ}$ \\
last follow-up & $11.9^{\circ} \pm 2.2^{\circ}$ \\
\hline
\end{tabular}

provide 1- or 2-level interbody fusion. The mean blood loss of $93.1 \mathrm{ml}$ and the mean hospital stay of 2.4 days demonstrate that this approach, if effective, is likely to cause substantially less morbidity than a dorsal approach to the spine. A standard posterior revision surgery would involve a laminectomy with potential concomitant fusion and extension of instrumentation. Such an approach is substantially more complicated, carrying a higher rate of CSF leakage and requiring the management of previously implanted spinal instrumentation. Another advantage to this approach relates to the fact that the posterior spinal elements, including the rostral facet joint capsules, are not disrupted through a lateral approach; thus, additional degeneration at the supraadjacent level may also be less likely to occur, as has been seen with ALIF. The use of this approach after a previous lateral fusion may be more problematic through the same side, but the contralateral side could be used.

The degree of subsidence in our study was less than in previous investigations. Marchi et al. reported, at 12 months, that $70 \%$ of 61 patients with minimally invasive lateral interbody fusion had $0 \%-49 \%$ subsidence and $30 \%$ had $50 \%-100 \%$ subsidence. ${ }^{10}$ Subsidence was typically seen at 6 weeks or earlier, and it occurred predominantly (68\%) in the inferior endplate. One might expect subsidence rates to be higher in ASD cases, because of the rigid caudal fusion, but this was not demonstrated in our study. The presence of pedicle screws in the vertebral body below may have been responsible for this finding. Careful attention to proper endplate preparation without violation of the cortical endplate is also critical to minimizing settling.

However, note that this procedure does not significantly add or improve global lumbar lordosis, as demonstrated by a slight loss of global lumbar lordosis after lateral surgery. Thus, its application in patients who have the potential for sagittal imbalance must be carefully considered. In addition, because of concerns regarding cage settling, these cases were carefully selected to exclude patients with any significant kyphosis, listhesis, or osteoporosis.

There are several limitations to this study. The first relates to the ability to generalize the results. With regard to the relief of neurological symptoms of stenosis, the minimally invasive lateral approach relies entirely on indirect decompression by elevating intervertebral column height, which expands the neuroforamen and tensions the longitudinal ligaments to open the central canal. While we found excellent clinical results, one might expect this approach to be occasionally inadequate in cases of severe stenosis; one of our patients underwent a subsequent formal neural decompression. A larger study with a wider variety of specific pathologies would be helpful to validate this technique across the broad spectrum of ASD.

Another major limitation relates to our ability to ascertain definitive fusion. The current gold standard for spinal fusion is direct inspection and palpation at surgery. Imaging surrogates, such as dynamic radiographs, 3D CT scans, and nuclear medicine studies, can be helpful. In this series we used sagittal and coronal reconstructed fine-cut CT scans to identify bridging bone between the treated vertebral bodies as the determinant of fusion, which is the best early indicator of successful arthrodesis. However, a follow-up longer than 2 years would also be helpful, as an osseous nonunion would likely become more apparent clinically or radiographically over more protracted periods of time. Longer follow-up studies are therefore necessary as well.

Finally, the application of rhBMP-2 in this setting represents an off-label FDA use. To date, however, few reports have indicated that this application is problematic through a direct lateral approach. This is in contradistinction to BMP use in transforaminal interbody fusion where the incidence of radiculitis has been reported to be as high as $11.4 \% .^{11}$ This difference is likely attributable to the fact that there is no direct exposure or contact with the spinal canal, dura mater, or neural elements. The use of BMP in this setting is thus more similar to its use in ALIF, which is the on-label indication for rhBMP-2.

\section{Conclusions}

This limited study suggests that minimally invasive lateral interbody fusion may be a viable alternative for treating ASD following a previous lumbosacral fusion. The approach can result in adequate indirect neural decompression as well as solid arthrodesis in the majority of cases without requiring supplemental posterior fixation.

\section{Disclosure}

Dr. Wang is a consultant for and receives royalty payments from DePuy Spine, Inc., and he is a consultant for Aesculap Spine.

Author contributions to the study and manuscript preparation include the following. Conception and design: Wang. Acquisition of data: Wang, Vasudevan. Analysis and interpretation of data: Vasudevan, Mindea. Drafting the article: Wang. Critically revising 
script: Wang.

\section{References}

1. Burkus JK, Transfeldt EE, Kitchel SH, Watkins RG, Balderston RA: Clinical and radiographic outcomes of anterior lumbar interbody fusion using recombinant human bone morphogenetic protein-2. Spine (Phila Pa 1976) 27:2396-2408, 2002

2. Cahill KS, Martinez JL, Wang MY, Vanni S, Levi AD: Motor nerve injuries following the minimally invasive lateral transpsoas approach. Clinical article. J Neurosurg Spine 17: 227-231, 2012

3. Cho W, Sokolowski MJ, Mehbod AA, Denis F, Garvey TA, Perl J, et al: MRI measurement of neuroforaminal dimension at the index and supradjacent levels after anterior lumbar interbody fusion: a prospective study. Clin Orthop Surg 5: 49-54, 2013

4. Cummock MD, Vanni S, Levi AD, Yu Y, Wang MY: An analysis of postoperative thigh symptoms after minimally invasive transpsoas lumbar interbody fusion. Clinical article. J Neurosurg Spine 15:11-18, 2011

5. Dakwar E, Cardona RF, Smith DA, Uribe JS: Early outcomes and safety of the minimally invasive, lateral retroperitoneal transpsoas approach for adult degenerative scoliosis. Neurosurg Focus 28(3):E8, 2010

6. Elowitz EH, Yanni DS, Chwajol M, Starke RM, Perin NI: Evaluation of indirect decompression of the lumbar spinal canal following minimally invasive lateral transpsoas interbody fusion: radiographic and outcome analysis. Minim Invasive Neurosurg 54:201-206, 2011

7. Isaacs RE, Hyde J, Goodrich JA, Rodgers WB, Phillips FM: A prospective, nonrandomized, multicenter evaluation of extreme lateral interbody fusion for the treatment of adult degenerative scoliosis: perioperative outcomes and complications. Spine (Phila Pa 1976) 35 (26 Suppl):S322-S330, 2010

8. Kepler CK, Sharma AK, Huang RC, Meredith DS, Girardi FP, Cammisa FP Jr, et al: Indirect foraminal decompression after lateral transpsoas interbody fusion. Clinical article. J Neurosurg Spine 16:329-333, 2012
M. Y. Wang, R. Vasudevan, and S. A. Mindea

9. Khan IS, Sonig A, Thakur JD, Bollam P, Nanda A: Perioperative complications in patients undergoing open transforaminal lumbar interbody fusion as a revision surgery. Clinical article. J Neurosurg Spine 18:260-264, 2013

10. Marchi L, Abdala N, Oliveira L, Amaral R, Coutinho E, Pimenta L: Radiographic and clinical evaluation of cage subsidence after stand-alone lateral interbody fusion. Clinical article. J Neurosurg Spine 19:110-118, 2013

11. Mindea SA, Shih P, Song JK: Recombinant human bone morphogenetic protein-2-induced radiculitis in elective minimally invasive transforaminal lumbar interbody fusions: a series review. Spine (Phila Pa 1976) 34:1480-1485, 2009

12. Ozgur BM, Aryan HE, Pimenta L, Taylor WR: Extreme Lateral Interbody Fusion (XLIF): a novel surgical technique for anterior lumbar interbody fusion. Spine J 6:435-443, 2006

13. Parker SL, Shau DN, Mendenhall SK, McGirt MJ: Factors influencing 2-year health care costs in patients undergoing revision lumbar fusion procedures. Clinical article. J Neurosurg Spine 16:323-328, 2012

14. Radcliff K, Kepler CK, Jakoi A, Sidhu GS, Rihn J, Vaccaro AR, et al: Adjacent segment disease in the lumbar spine following different treatment interventions. Spine J 13:13391349,2013

15. Smorqick Y, Baker K, Bachison C, Herkowith H, Montgomery D, Fischgrund J: Hidden blood loss during posterior spine fusion surgery. Spine J 13:877-881, 2013

16. Tohmeh A, Rogers W, Peterson M: Dynamically evoked, discrete-threshold electromyography in the extreme lateral interbody fusion approach. Clinical article. J Neurosurg Spine 14:31-37, 2011

Manuscript submitted September 18, 2013.

Accepted August 22, 2014.

Please include this information when citing this paper: published online October 10, 2014; DOI: 10.3171/2014.8.SPINE13841.

Address correspondence to: Michael Y. Wang, M.D., Lois Pope Life Center, Department of Neurological Surgery, 1095 NW 14th Terrace, Miami, FL 33136. email: mwang2@ med.miami.edu. 